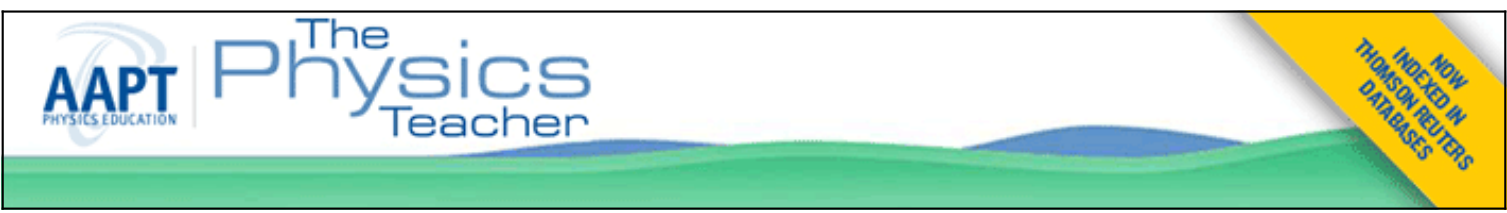

\title{
A New Lecture-Tutorial for Teaching about Molecular Excitations and Synchrotron Radiation
}

Colin S. Wallace, Edward E. Prather, Seth D. Hornstein, Jack O. Burns, Wayne M. Schlingman, and

Timothy G. Chambers

Citation: The Physics Teacher 54, 40 (2016); doi: 10.1119/1.4937972

View online: http://dx.doi.org/10.1119/1.4937972

View Table of Contents: http://scitation.aip.org/content/aapt/journal/tpt/54/1?ver=pdfcov

Published by the American Association of Physics Teachers

\section{Articles you may be interested in}

Teaching Physics with Music

Phys. Teach. 53, 415 (2015); 10.1119/1.4931010

Light-Emitting Diodes: Learning New Physics

Phys. Teach. 53, 210 (2015); 10.1119/1.4914558

The graduate research field choice of women in academic physics and astronomy: A pilot study AIP Conf. Proc. 1513, 66 (2013); 10.1063/1.4789653

A new multimedia resource for teaching quantum mechanics concepts

Am. J. Phys. 80, 148 (2012); 10.1119/1.3657800

To a physicist new to teaching astronomy

Phys. Teach. 35, 172 (1997); 10.1119/1.2344630

\section{Collect Clean, Repeatable,} and Noise-Resistant

Motion Data

Vernier Dynamics Cart and Track System with Motion Encoder

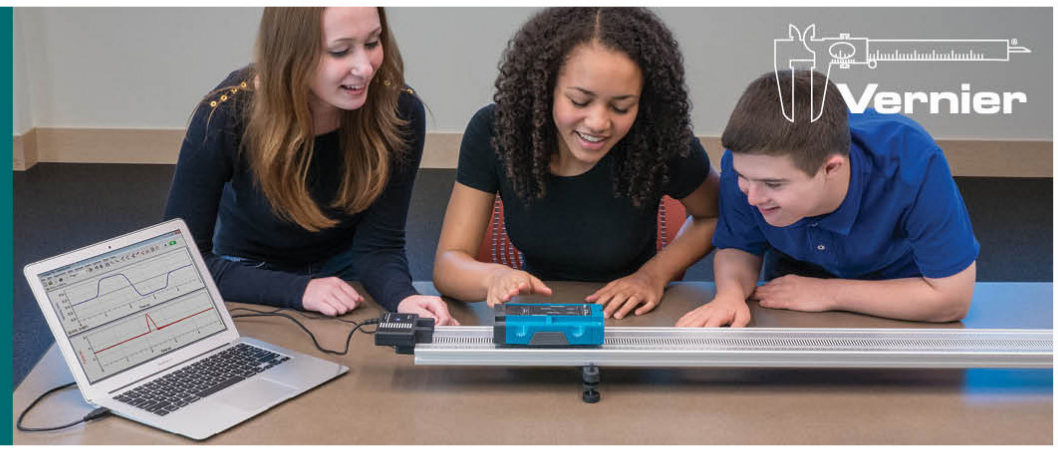




\title{
A New Lecture-Tutorial for Teaching about Molecular Excitations and Synchrotron Radiation
}

\author{
Colin S. Wallace, University of North Carolina at Chapel Hill, Chapel Hill, NC \\ Edward E. Prather, University of Arizona, Tucson, AZ \\ Seth D. Hornstein and Jack O. Burns, University of Colorado Boulder, Boulder, CO \\ Wayne M. Schlingman, The Ohio State University, Columbus, $\mathrm{OH}$ \\ Timothy G. Chambers, University of Michigan, Ann Arbor, MI
}

\section{I}

ight and spectroscopy are among the most important and frequently taught topics in introductory college-

level general education astronomy courses (hereafter Astro 101). ${ }^{1}$ This is due to the fact that the vast majority of observational data studied by astronomers arrives at Earth in the form of light. While there are many processes by which matter can emit and absorb light, Astro 101 courses typically limit their instruction to the Bohr model of the atom and electron energy level transitions. In this paper, we report on the development of a new Lecture-Tutorial to help students learn about other processes that are responsible for the emission and absorption of light, namely molecular rotations, molecular vibrations, and the acceleration of charged particles by magnetic fields. Note that this paper primarily focuses on describing the variety of representations and reasoning tasks designed for this Lecture-Tutorial; while the end of this paper highlights some data that are suggestive of the LectureTutorial's effectiveness, our more comprehensive analysis of its efficacy will be presented in a future publication.

The design and development process for this new LectureTutorial followed the principles and methodology that guided the development of the original Lecture-Tutorials for Introductory Astronomy. ${ }^{2}$ Each of the 44 previously developed evidence-based Lecture-Tutorials is a two- to six-page worksheet containing carefully sequenced and conceptually scaffolded Socratic-style questions, utilizing multiple representations of scientific information, that actively engage collaborative student learning groups in constructing more expert-like understandings of a small number of closely related astrophysical topics. Several prior research studies show that the implementation of Lecture-Tutorials can help students significantly increase their understandings of difficult physics and astronomy concepts beyond what they typically achieve during traditional lecture-based instruction. ${ }^{3}$

The development of this Lecture-Tutorial was one of the initial projects of a new collaboration between scientists and science education researchers from Associated Universities, Inc. (AUI), the Center for Astronomy Education (CAE) at the University of Arizona (UA), the University of Colorado Boulder (CU-Boulder), and the University of North Carolina at Chapel Hill (UNC). One of the goals of this collaboration is to create the tools STEM instructors need to transform their college classes into active-engagement, collaborative learning environments using foundational and cutting-edge radio astronomy topics. For this particular Lecture-Tutorial, our goals were to help students understand:

1) the interactions between light and matter involving molecular rotations, molecular vibrations, and synchrotron radiation;

2) the wavelengths of light and types of spectra that are produced by these different processes;

3) the connection between these processes and real astrophysical phenomena; and

4) the telescopes whose wavelength sensitivities are best suited for observing each particular type of matter-energy interaction.

\section{A new Lecture-Tutorial on molecular vibrations, molecular rotations, and syn- chrotron radiation}

During the prior research and development on the Lecture-Tutorial that focused on the Bohr model of the atom, we found that many students leave a traditional lecture unable to consistently and coherently reason about whether an electron moving from a higher to lower energy level (and vice versa) results in the emission or absorption of light, and how the wavelength of the emitted/absorbed light is related to the energy difference between the electron's initial and final states. We were therefore not surprised to find that students exhibit similar difficulties when asked to reason about how changes in the rotational or vibrational energy state of a molecule are related to the emission or absorption of light. As a result, we begin the new Lecture-Tutorial with questions that help students develop their conceptual understandings of, and abilities to reason about, the emission and absorption of different wavelengths of light via molecular vibrations and rotations.

Note that throughout this new Lecture-Tutorial we explicitly use many different representations. We hereafter use the term "pedagogical discipline representations" (PDRs) to refer to the body of representations that are useful for teaching and learning about a given discipline, such as astronomy, even though those representations may not be typically used in 
A molecule can change its vibrational energy state by emitting or absorbing an infrared photon. In each of the four cases shown below (A-D), a molecule is shown transitioning between two different energy states depending on whether the molecule absorbed or emitted a photon.

A

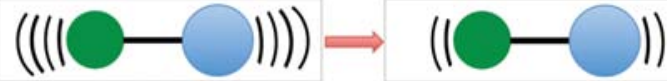

Before

B

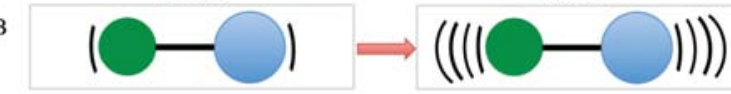

Before

C

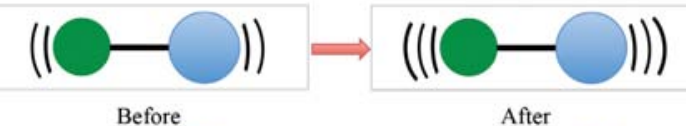

D
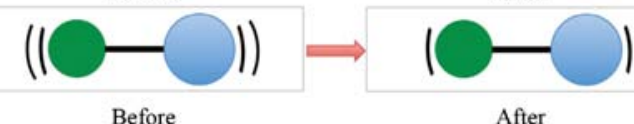

After

1) Which of the cases (A-D) shown above correspond with the absorption of light and which correspond with the emission of light? Draw in a squiggly arrow representing a photon with the appropriate direction for each case (A-D)

2) In which case was a photon with the longest wavelength absorbed? Explain your reasoning

3) In which case was a photon with the greatest energy emitted? Explain your reasoning.

Fig. 1. Sample questions from the Lecture-Tutorial that help develop students' understandings of the emission and absorption of light by molecular vibrations.

textbooks or by two experts in a field that are communicating with one another. Different PDRs may have complementary abilities to deepen students' understandings due to the fact that these representations have different scientific and educational affordances. ${ }^{4}$ Consequently, when we develop a new Lecture-Tutorial, we use the widest variety of PDRs possible, including graphs, equations, pictures, and tables of data, so that we may engage the critical thinking skills of the largest possible range of students and help them become more fluent in the science content they are studying. For example, in the Lecture-Tutorial that focuses on the Bohr model of the atom, students reason about a combination of pictorial and word-based representations of photon and atom interactions, electron energy levels, and the relationship between energy level transitions and the wavelength and energy of emitted/ absorbed light. The new Lecture-Tutorial uses carefully chosen PDRs designed to help students envision the physical behavior of molecules that change energy states due to the emission and absorption of photons, and how these matterenergy interactions relate to different representations of spectra. Figures 1-5 show excerpts from the Lecture-Tutorial that highlight the range of question types, reasoning tasks, and representations used; the full Lecture-Tutorial contains additional questions that aid in the transition between the questions shown in this paper. As shown in Fig. 1, we use the number of arcs on either side of a molecule to qualitatively represent its vibrational energy state. More arcs correspond to a vibrational state of greater energy. Figure 2 shows our use of arrows to qualitatively represent the rotational energy state of a molecule. Longer arrows correspond to a rotational

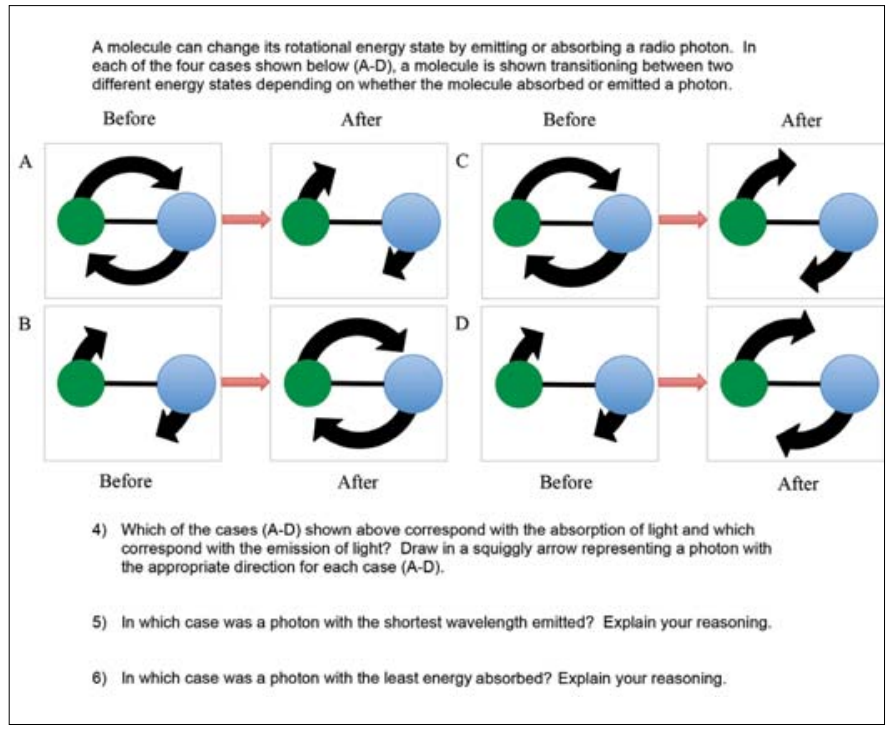

Fig. 2. Sample questions from the Lecture-Tutorial that help develop students' understandings of the emission and absorption of light by molecular rotations.

state of greater energy. Through field tests with over 1000 students, we found that these arrows and arcs facilitate students' discourse about changes in rotational and vibrational energy states, and, as a result, students are able, post-instruction, to incorporate these pictorial representations into explanations that are more scientifically coherent than those provided after traditional lecture-based instruction.

Figures 1 and 2 also show a sequence of questions used in the new Lecture-Tutorial to engage students about how changes in a molecule's vibrational or rotational energy state correspond to the emission or absorption of a photon. The new Lecture-Tutorial requires students to reason about how the energy/wavelength/frequency of a photon that is emitted or absorbed as a result of one molecular energy state transition compares to the energy/wavelength/frequency of a photon emitted or absorbed due to a different energy state transition. For example, students are expected to realize that both Case B and C in Fig. 1 correspond to the absorption of a photon, although the photon absorbed in Case $\mathrm{C}$ must have a longer wavelength (or lower energy) than the photon absorbed in Case B. Reasoning tasks associated with the energy/ wavelength/frequency of an emitted/absorbed photon target and reinforce conceptual ideas and reasoning pathways that have been developed in other Lecture-Tutorials.

Section II of the new Lecture-Tutorial is devoted to synchrotron radiation. It focuses on helping students understand that synchrotron radiation has a continuous spectrum that is not dependent on temperature (i.e., it is non-thermal). Figure 3 shows a question from this section asking students to sketch a qualitatively correct spectrum for the light produced by a pulsar whose surface emits a thermal radiation and whose jets emit synchrotron radiation.

Section III requires students to synthesize all the ideas covered in Sections I and II and apply them to real astrophysical contexts. Figure 4 shows a question from this section 


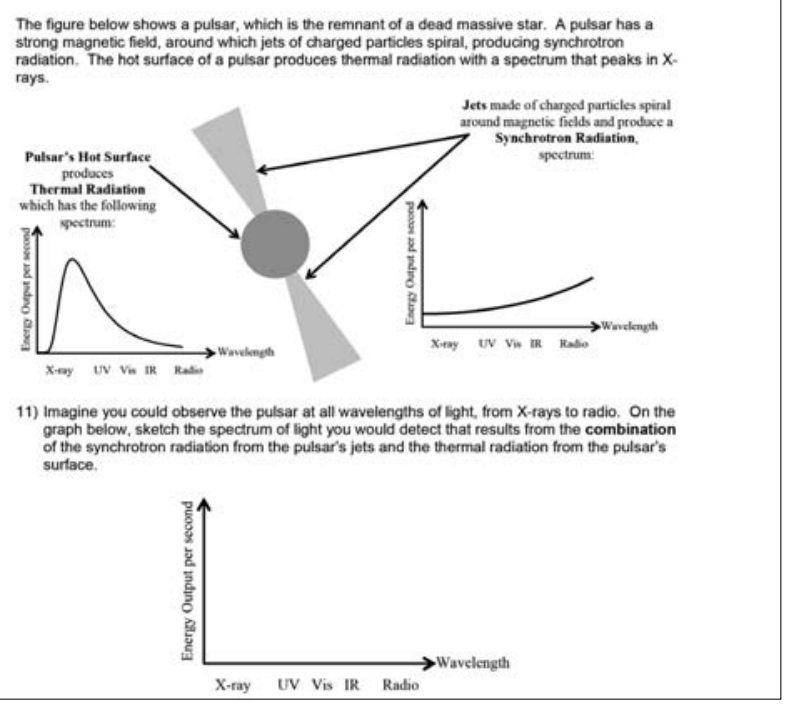

Fig. 3. Sample question from the Lecture-Tutorial that asks students to reason about a combined thermal and synchrotron spectrum.

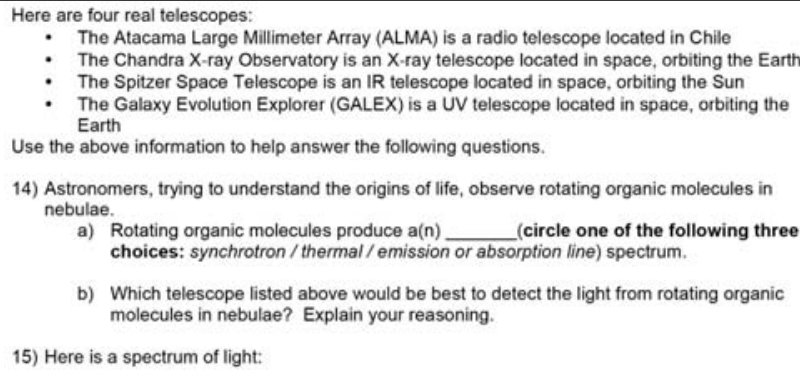

b) Which telescope listed above would be best to detect the light from rotating organic molecules in nebulae? Explain your reasoning.

15) Here is a spectrum of light:

Fig. 4. Sample questions from the Lecture-Tutorial that require students to select the appropriate telescope based on what they have learned from earlier sections of the Lecture-Tutorial.

that asks students to select the appropriate telescope needed to make a real astronomical observation. To correctly answer this question, a student must recognize that the rotating organic molecules will emit in the radio and produce a line spectrum. The Atacama Large Millimeter Array (ALMA) is the only radio telescope listed, so it is the only one out of the four possible telescopes that can be used to observe this spectrum. Figure 5 shows the Lecture-Tutorial's culminating question, which requires students to evaluate multiple linked representations and to use their knowledge of molecular vibrations, molecular rotations, and synchrotron radiation to re-represent all incorrect information. To complete this task, students must recognize that 1 ) the vibrational energy state transition depicted would lead to the emission, not absorption, of a photon, 2) the photon would not be in the radio, since vibrational energy state transitions generally produce

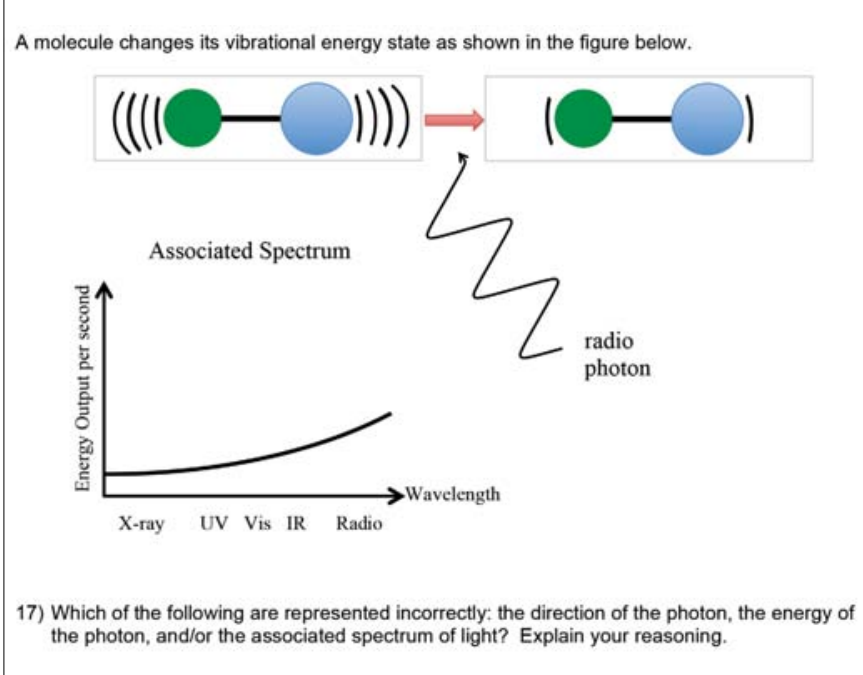

Fig. 5. Sample question from the Lecture-Tutorial that requires students to identify each problem with the above picture.

infrared light, and 3) the spectrum shown is incorrect, since it depicts the continuous spectrum of synchrotron radiation and not the line spectrum of vibrational energy state transitions.

\section{Assessment}

In order to gain insight into the effectiveness of this new Lecture-Tutorial, we created a survey of 12 conceptually challenging questions. These questions probe the same content as the Lecture-Tutorial, although they differ in their context and/or format from the questions asked on the Lecture-Tutorial itself. Figure 6 shows a subset of these questions. The assessment's face validity and content accuracy were established by the review of content experts in radio astronomy.

We pilot tested this new Lecture-Tutorial with over 1000 students over three semesters at UA and CU-Boulder. Over these three semesters, we designed many different assessment methodologies in order to measure the effects of different implementations of this Lecture-Tutorial.

For this publication, we report the data we gathered from only one of the many methodologies for assessing the efficacy of the Lecture-Tutorial; results from our larger dataset will appear in a future publication. In the spring of 2014, we tested the implementation of the Lecture-Tutorial by examining the understandings of a population of students enrolled in two sections of Astro 101 at CU-Boulder. Both sections had similar demographics and distributions of overall grades. The two sections each received a carefully crafted lecture from the same instructor on the relevant astrophysics of each concept before doing the Lecture-Tutorial. One section of this Astro 101 course took the 12-question assessment before any instruction on the topics of the Lecture-Tutorial (pre-test). This section of the course also took the 12-question assessment after the lecture (post-lecture) but before they worked through the Lecture-Tutorial activity (pre-Lecture-Tutorial). The other 


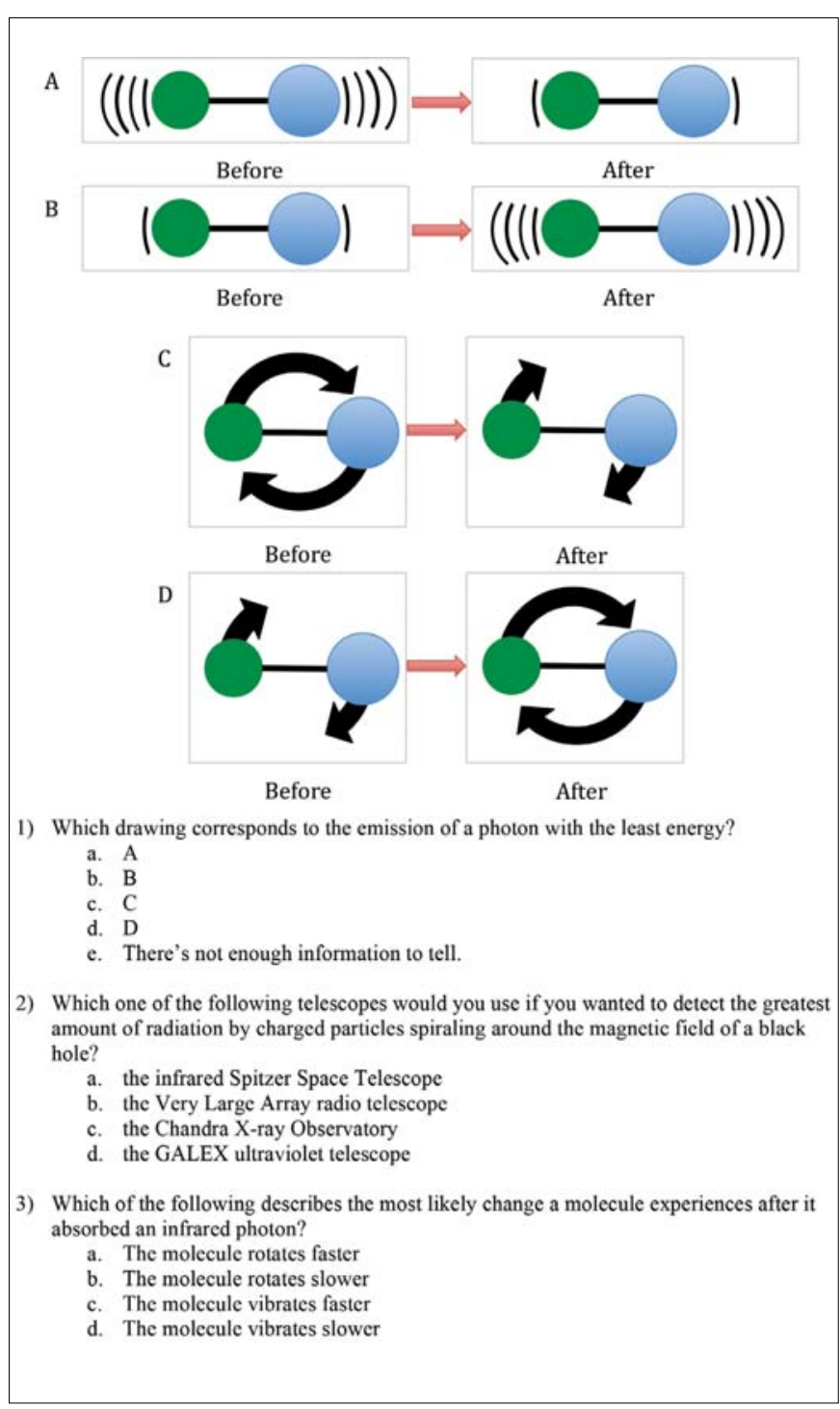

Fig. 6. Sample questions assessment used to measure the efficacy of the Lecture-Tutorial.

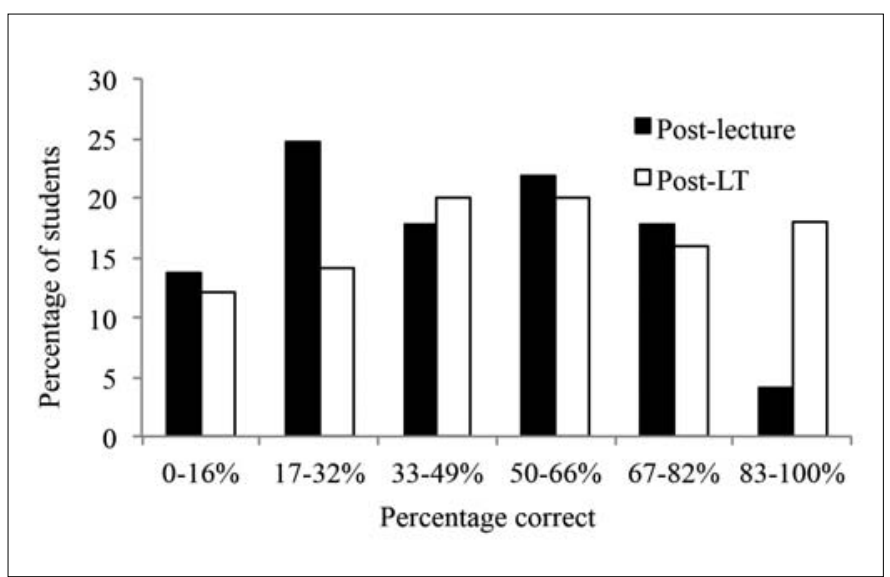

Fig. 7. Histograms of the post-lecture and post-Lecture-Tutorial populations binned by percentage correct on the post-test.

section did not take a pre-test but did take the assessment after completing the Lecture-Tutorial activity (post-Lecture-Tutorial); note that the students in this section were also asked two low-level Think-Pair-Share questions during lecture. The pre-Lecture-Tutorial and post-Lecture-Tutorial results from these two Astro 101 sections were each compared to the pretest results. This research design allows us to isolate the improvement in understanding achieved from lecture alone and compare this result with the amount of gain in understanding achieved when the active engagement Lecture-Tutorial is done in class immediately following lecture.

The single section that took the 12-question assessment as a pre-test had an average pre-test score of $22 \%$ correct $(n=$ 112). This pre-test score is equal to the other pre-test scores from our larger dataset, in which Astro 101 populations at multiple institutions who received no prior instruction on these topics also had an average score on this assessment of $22 \%$ correct. We therefore assume a pre-test score of $22 \%$ for the other spring 2014 Astro 101 section at CU-Boulder, for which we did not administer the pre-test. By not administering the pre-test to this population, we also ensure that the students who took the assessment post-Lecture-Tutorial had never seen any of the 12 conceptual questions used in this study (this is not true for the post-lecture section in our comparison below).

The average correct score for the students post-lecture was $49 \%(n=73)$. The average correct score for the students post-Lecture-Tutorial was $57 \%(n=50)$. These results suggest that a well-focused lecture may indeed help students improve their understanding of these topics (pre- to post-lecture gain of 27\%). Additionally, through the implementation of this new Lecture-Tutorial, which takes only 15 minutes (approximately) of class time, a class may experience an additional increase in understanding (post-lecture to postLecture-Tutorial gain of $8 \%$ ). This result is consistent with the gains in students' understandings on other astrophysics topics achieved by incorporating other Lecture-Tutorials into Astro 101 courses. ${ }^{3} \mathrm{~A} z$-test comparing the post-lecture and post-Lecture-Tutorial means result in a $p$-value of $p=0.06$. This suggests that the differences between the post-lecture and post-Lecture-Tutorial populations would be produced by chance only $6 \%$ of the time.

Further evidence for the effectiveness of the LectureTutorial can be seen in Fig. 7, which shows the percentage of students in both populations in bins based on the percentage correct on the post-test. Note that the post-lecture distribution has a greater concentration of scores at the lower end of the distribution (a range of scores that also happens to include the pre-instruction average) than does the post-Lecture-Tutorial group. Overall, this analysis suggests that the new Lecture-Tutorial may be effective at moving a subset of students to a higher level of understanding, which they may not have achieved after lecture alone. This result is consistent with the levels of understanding students have been shown to achieve after completing other Lecture-Tutorials; as noted above, we will continue to investigate the effectiveness of different implementations of this Lecture-Tutorial in future publications. 
Physics teachers... get your students registered for the preliminary exam in the U.S. Physics Team selection process.

\section{All physics students are encouraged to participate in the American Association of Physics Teachers' Fnet=ma Contest!}

The Fnet=ma Contest is the United States Physics Team selection process that leads to participation in the 47th Annual International Physics Olympiad (IPh0) in Zurich, Switzerland, July 10-18, 2016. The U.S. Physics Team Program provides a oncein-a-lifetime opportunity for students to enhance their physics knowledge as well as their creativity, leadership, and commitment to a goal.

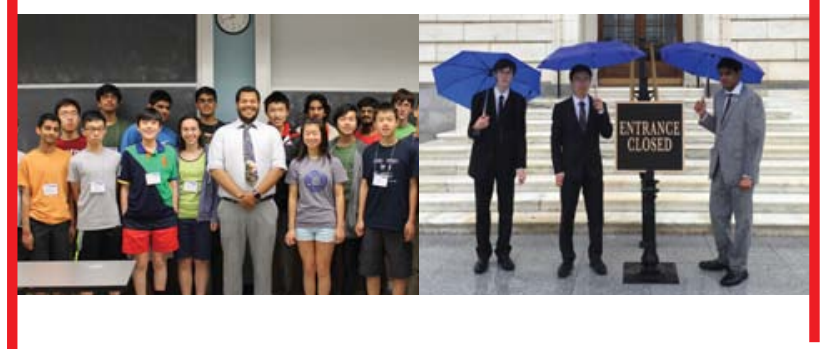

School Fee: \$35 per school (\$25 fee for teachers who are AAPT members) plus $\$ 4$ per student for WebAssign or \$8 per student for PDF download. Two or more teachers from the same school pay only one school fee.

For program information and registration visit: http://www.aapt.org/physicsteam (Registration for 2016 competition ends Dec. 14, 2015)

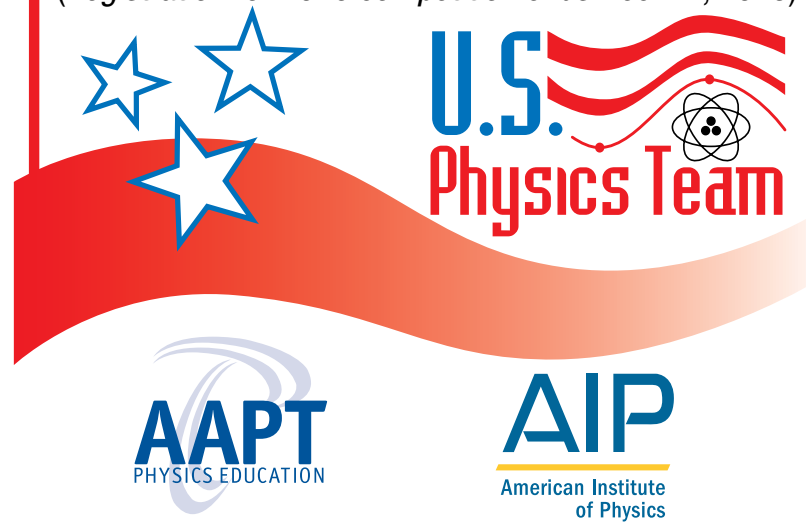

\section{Availability of the Lecture-Tutorial}

Instructors who wish to obtain a copy of the current version of the Lecture-Tutorial, as well as the accompanying lecture slides and Think-Pair-Share ("clicker") questions that have been developed to support this Lecture-Tutorial, should contact the authors. A copy of the Lecture-Tutorial is also included as an online appendix. ${ }^{5}$

\section{Acknowledgments}

This work was made possible by the generous support of Associated Universities, Inc. (AUI). We would particularly like to thank Ethan Schreier, John Mester, John Stoke, and Timothy Spuck for their guidance and support. Additionally, we would like to thank Johanna Teske and Jeffrey Eckenrode for their help with the pilot testing and data collection of this curriculum.

\section{References}

1. T. F. Slater, J. P. Adams, G. Brissenden, and D. Duncan, "What topics are taught in introductory astronomy courses?" Phys. Teach. 39, 52-55 (Jan. 2001).

2. E. E. Prather, T. F. Slater, J. P. Adams, and G. Brissenden, Lecture-Tutorials for Introductory Astronomy, 3rd ed. (Pearson Education, Inc., San Francisco, CA, 2013).

3. E. E. Prather, T. F. Slater, J. P. Adams, J. M. Bailey, L. V. Jones, and J. A. Dostal, "Research on a lecture-tutorial approach to teaching introductory astronomy for non-science majors," Astron. Educ. Rev. 3, 122-136 (2004); E. E. Prather, and G. Brissenden, "Clickers as data gathering tools and students' attitudes, motivations, and beliefs on their use in this application," Astron. Educ. Rev. 8, 010103 (2009); C. S. Wallace, E. E. Prather, and D. K. Duncan, "A study of general education astronomy students' understandings of cosmology. Part V. The effects of a new suite of cosmology lecture-tutorials on students' conceptual knowledge," Int. J. Sci. Educ. 34, 1297-1314 (2012); M. C. LoPresto and S. R. Murrell, "Using the Star Properties Concept Inventory to compare instruction with lecture tutorials to traditional lectures," Astron. Educ. Rev. 8, 010105 (2009).

4. C. Linder, "Disciplinary discourse, representation, and appresentation in the teaching and learning of science," Eur. J. Sci. Math. Educ. 1, 657-666 (2013); T. Fredlund, C. Linder, J. Airey, and A. Linder, "Unpacking physics representations: Towards an appreciation of disciplinary affordance," Phys. Rev. ST: Phys. Educ. Res. 10, 020129 (2014).

5. The Lecture-Tutorial can be downloaded from TPT Online at http://dx.doi.org/10.1119/1.4937972.

University of North Carolina at Chapel Hill, Chapel Hill, NC 27599; cswphys@email.unc.edu 